\title{
Genetic testing in asymptomatic minors: recommendations of the European Society of Human Genetics
}

\author{
Recommendations of the European Society of Human Genetics
}

European Journal of Human Genetics (2009) 17, 720-721; doi:10.1038/ejhg.2009.26; published online 11 March 2009

\section{Introductory considerations}

Since their introduction, genetic tests and their widespread application have been considered carefully, with special attention to the release of information about the test and test results, the confidentiality of genetic information, whether the test was taken voluntarily, the responsibility towards blood relatives and the psychological impact of the test. When, for example, a genetic disorder is diagnosed in an index-patient, other family members might also be at risk of developing the disease or passing it to their offspring. For competent adults, it is agreed that the provision of genetic testing should be based on respect for the principle of selfdetermination of the persons concerned. For this reason, any genetic testing, even when offered systematically (eg as a screening test), should be subject to their express, free and informed consent. In this set of recommendations genetic screening of asymptomatic minors is not considered, except for the possibility of incidental discovery of carrier status. Neither will susceptibility testing for multifactorial diseases be considered, owing to its limited clinical validity and utility at this time. These recommendations concern genetic testing of asymptomatic minors in a clinical context (testing on request of the parents or the minors themselves).

Careful consideration is needed when family members at risk are asymptomatic children or adolescents. Cautious reflection is needed on whether and under what conditions genetic testing might be performed on asymptomatic minors. With these recommendations, the European Society of Human Genetics (ESHG) wants to address in detail the issue of genetic testing in asymptomatic minors. We wish to stress that predictive genetic testing of asymptomatic minors should only be considered after detection of the mutation

*Correspondence: Dr P Borry, Center for Biomedical Ethics and Law, Katholieke Universiteit Leuven, Kapucijnenvoer 35/3, Box 7001, Leuven 3000, Belgium.

E-mail: Pascal.Borry@med.kuleuven.be in the family. Of course, diagnostic testing of minors can follow clinical assessment to confirm a diagnosis.

First, these recommendations will propose some general considerations regarding the treatment of minors and the process of genetic counseling. Second, recommendations are formulated regarding presymptomatic and predictive genetic testing in minors, and for incidental and intentional carrier testing. We are aware of the terminological discussion with regard to genetic testing, but cannot study this in detail in this document. For the purpose of this document, we will use following definitions. The term presymptomatic diagnosis is only used for those situations in which an abnormal test result will almost inevitably lead to development of the disease at some point in later life, whereas the term predictive testing covers a broader range of situations, namely also situations in which an abnormal test result implies a substantial risk, but not certainty, of developing the disease later in life. In addition, it is essential to distinguish between presymptomatic and predictive genetic testing for monogenic diseases and susceptibility genetic testing for multifactorial diseases. Carrier tests are performed to determine whether an asymptomatic male or female carries a mutation relevant for an autosomal recessive disorder, whether an asymptomatic female carries a mutation relevant for an $\mathrm{X}$-linked disorder or whether an asymptomatic person has a balanced chromosomal rearrangement. Minors are defined as all human beings who have not reached the age of legal majority in health decisions. The onset of a condition is defined as the time of appearance of the first clinical symptoms of the condition or its first manifestations detected by laboratory tests, radiological results or other technical examinations.

To discuss these issues and produce recommendations from the professional point of view, a draft background document and recommendations were prepared by Pascal Borry and Kris Dierickx, who had been involved in a EUROGENTEST (www.eurogentest.org) work package on testing in minors. These documents were discussed in a 
workshop with an ad-hoc committee of the Public and Professional Policy Committee (PPPC) of the ESHG at a workshop in November 2007 in Leuven, Belgium. Revised versions were discussed at the PPPC meetings in April 2008 in Amsterdam and in June 2008 in Barcelona. The recommendations, as endorsed by the ESHG board, are given below, and the background considerations are given separately, in the subsequent document.

\section{Recommendations}

\section{General considerations}

(1) The primary reason to carry out a genetic test on a person who does not have the capacity to consent should be his or her direct benefit.

(2) The opinion of the minor shall be taken into consideration as an increasingly determining factor in proportion to his or her age and degree of maturity. Decision-making involving the health care of a minor should include, to the greatest extent feasible, his or her consent or assent.

(3) The parents (or legal guardians) should participate as much as possible in the decision-making process regarding the health care of their children. If the decision of the minor's parents or legal guardians is not to the direct benefit of the minor, health care professionals have the responsibility to defend the interests of the minor.

(4) Although parental support is recommended, asymptomatic minors with a genetic risk who are well informed, have an adequate understanding of a test and its potential implications, have the capacity to make this decision, are not exposed to external pressure and have received appropriate counseling, should be considered competent to undergo genetic testing.

(5) Parents are responsible for informing their children about their genetic risk, tailoring the information to their age-related capacities. Genetic services should take a supportive role in this process.

(6) Genetic counseling is always required when considering genetic testing in asymptomatic minors. Health care professionals should provide sufficient attention to the anxieties and concerns of the parents and the children not only in relation to genetic testing, but also in dealing and coping with the outcomes.

\section{Presymptomatic and predictive genetic testing}

(7) Presymptomatic and predictive genetic testing of minors for conditions with adult-onset is acceptable only if preventive actions (eg preventive surgery or early detection aimed at therapeutic interventions) can be initiated before adulthood. Otherwise presymptomatic and predictive genetic testing in minors for adult-onset disorders should be deferred until the person has the maturity and competence to understand the nature of the decision and its implications.

(8) In the case of presymptomatic and predictive genetic testing for conditions which become manifest in childhood and which can be effectively treated or prevented, there are good reasons to comply or to actively bring up the possibility of a test. However, if the preventive and therapeutic measures will be deferred to a later time, the justification for immediate testing is less compelling and careful, supportive counseling will often be appropriate whether or not testing happens at that time.

(9) In the case of presymptomatic and predictive genetic testing for conditions which become manifest in childhood and which can not be effectively treated or prevented, there are both benefits and risks, and usually neither the benefits nor the risks completely outweigh each other. Genetic testing could be considered if this would be to the psychological or social benefit of the child and his family.

\section{Intentional carrier testing}

(10) Testing for carrier status should be discouraged until the minor has the maturity and competence to understand the nature of the decision and its implications, and is able to consent. In some cases, intentional carrier testing of children might be considered in families in which other children within the family have been identified incidentally as carriers or have been identified as having the disorder.

\section{Incidental discovery of carrier status}

(11) The possibility of incidental discovery of carrier status should be discussed during the pre-test counseling. Parents should decide before the test whether they would like to receive such a result or not, and if they want to be informed of such a result. These should always be given during a post-test counseling session.

\section{The members of the Public and Professional Policy} Committee (PPPC) of ESHG were in 2007-2008

Martina Cornel (chair, Amsterdam, The Netherlands), Gerry Evers-Kiebooms (Leuven, Belgium), Ségolène Aymé (Paris, France), Suzanne Braga (Bern, Switzerland), Franca Dagna Bricarelli (Genoa, Italy), Shirley Hodgson (London, UK), Gyorgy Kosztolany (Pécs, Hungary), Jan Lubinski, (Szczecin, Poland), Meral Özgüc (Ankara, Turkey), Christine Patch (London, UK), Jorge Sequeiros (Porto, Portugal), Lisbeth Tranebjaerg (Copenhagen, Denmark) Veronica van Heyningen (Edinburgh, UK).

The workshop participants were Pascal Borry, Kris Dierickx, Gerry Evers-Kiebooms (all three from Leuven, Belgium), Martina Cornel (Amsterdam, The Netherlands) and Angus Clarke (Cardiff, UK). 